\title{
A FACILE SYNTHESIS OF POROUS HEMATITE NANOMATERIALS AND THEIR FAST SORPTION OF CR (VI) IN WASTEWATER
}

\author{
CHENGLIANG HAN ${ }^{a *}, b$ JINGSONG XIE ${ }^{a}$, CHONGHAI DENG ${ }^{a}$, DIFANG ZHAO \\ ${ }^{a}$ Department of Chemical and Material Engineering, Hefei University, Hefei 230022, P.R. China \\ ${ }^{b}$ Institute of Solid State Physics, Chinese Academy of Sciences, Hefei, 230031,P.R. China
}

(Received: September 5, 2011 - Accepted: July 12, 2012)

\begin{abstract}
Porous hematite $\left(\alpha-\mathrm{Fe}_{2} \mathrm{O}_{3}\right)$ nanomaterials can be simply acquired from amorphous iron (III) hydroxide $\left(\mathrm{Fe}(\mathrm{OH})_{3}\right)$ in neutral solution by an evaporation method in an open quartz flask in a vacuum drying oven. Results show that the as-obtained $\alpha-\mathrm{Fe}_{2} \mathrm{O}_{3}$ particles at $180^{\circ} \mathrm{C}$ for $2 \mathrm{~h}$ or so are nearly spindle in shape. Each of $\alpha-\mathrm{Fe}_{2} \mathrm{O}_{3}$ microspindles is made of many ultrafine $\alpha-\mathrm{Fe}_{2} \mathrm{O}_{3}$ nanoparticles and results in a porous structure. The as-prepared porous $\alpha$ - $\mathrm{Fe}_{2} \mathrm{O}_{3}$ powders have higher surface area $\left(\sim 78 \mathrm{~m}^{2} / \mathrm{g}\right)$ and can rapidly remove $\mathrm{Cr}(\mathrm{VI})$ ions in wastewater at room temperature. The model of the adsorption kinetic of $\mathrm{Cr}(\mathrm{VI})$ is best adapted to the pseudo second-order equation.
\end{abstract}

Keywords: Hematite, Thermal evaporation, Porous structure, Removal of $\mathrm{Cr}$ (VI)

\section{INTRODUCTION}

The design and synthesis of various morphologies nanostructures have been important research subjects in nanoscience and nanotechnology due to their unique size and shape-dependent chemical and physical properties. Nanosturctured hematite $\left(\alpha-\mathrm{Fe}_{2} \mathrm{O}_{3}\right)$ is a typical environmentally friendly semiconductor $\left(\mathrm{E}_{\mathrm{a}}=2.1 \mathrm{eV}\right)$ materials and has been extensively applied in the production of catalysts [1,2], gas sensors [3-5], photo-assisted electrolysis of water [6,7], and electromagnetic devices [8].

Environmental contamination by chromium is a major problem in industrialized areas. It is well known that $\mathrm{Cr}$ (VI) is more toxic than $\mathrm{Cr}$ (III) to animals and plants. Thus, the removal of $\mathrm{Cr}$ (VI) from wastewater is of significant importance from an environmental viewpoint. Various conventional techniques have been employed for treating $\mathrm{Cr}(\mathrm{VI})$-contaminated water such as chemical precipitation [9], redox reaction [10,11], membrane separation [12], ion exchange $[13,14]$ and adsorption [15]. Of these methods, precipitation is usually sludge production. Ion exchange and membrane are not economically attractive because of their high operating costs. Adsorption has emerged as a cost-effective technique for removing metals from wastewater and has been widely studied during recent decades.

Nanomaterials with novel morphologies and microstructures exhibited effective functions are promising for impurity removal because of their large specific surface areas and unique structures [16, 17]. For example, Many nanostructured materials such as $\mathrm{Y}_{2}(\mathrm{OH}){ }_{5} \mathrm{NO}_{3} .1 .5 \mathrm{H}_{2} \mathrm{O}$ and $\mathrm{Y}_{2} \mathrm{O}_{3}$ [18], $\mathrm{MnFe}_{2} \mathrm{O}_{4}$ [19], hydrous titanium (IV) oxide [20] and $\mathrm{Fe} @ \mathrm{Fe}_{2} \mathrm{O}_{3}$ nanomaterials [21] have been used for the removal of $\mathrm{Cr}$ (VI) ions in wastewater. However, the research on efficiency and sorpted quantity for $\mathrm{Cr}$ (VI) ions on cost-low $\alpha-\mathrm{Fe}_{2} \mathrm{O}_{3}$ absorbents are rarely reported. In this article, the porous $\alpha-\mathrm{Fe}_{2} \mathrm{O}_{3}$ nanomaterials were prepared and used to remove the $\mathrm{Cr}(\mathrm{VI})$ ions in wastewater.

\section{EXPERIMENTAL}

For porous $\alpha-\mathrm{Fe}_{2} \mathrm{O}_{3}$ synthesis: The porous hematite micropowders were prepared as follows. Firstly, the $\mathrm{Fe}(\mathrm{OH})_{3}$ precursors were precipitated from $\mathrm{FeCl}_{3} \cdot 6 \mathrm{H}_{2} \mathrm{O}$ solution by adding proper ammonia $\left(\mathrm{NH}_{4} \cdot \mathrm{H}_{2} \mathrm{O}\right)$. Then, the above $\mathrm{Fe}(\mathrm{OH})_{3}$ with the solution was transferred into to a $100 \mathrm{~mL}$ quartz flask. Finally, the above flask without a lid was placed in a vacuum drying oven and maintained at $180^{\circ} \mathrm{C}$ for $2 \mathrm{~h}$. After cooling to ambient temperature, the solid red powders in the flask were ultrasonically rinsed for several times in deionized water and ethanol, respectively, and finally collected by a centrifuge and dried in a vacuum oven at $80^{\circ} \mathrm{C}$ for $6 \mathrm{~h}$.

For samples characterizations: X-ray diffraction (XRD) patterns were measured on a Philips X'pert diffractometer using $\mathrm{CuK}_{\alpha}$ radiation $(0.15419$ $\mathrm{nm})$. Morphology of the products was observed on a Sirion 200 FEG field emission scanning electron microscope (FESEM). High resolution transmission electron microscopic (HRTEM) examination was conducted on a JEOL-2010 microscope attached with an energy-dispersive x-ray spectrometer (Oxford, Link ISIS). Nitrogen adsorption isotherms were measured at $77 \mathrm{~K}$ on Micrometrics ASAP 2020 equipment.
For $\mathrm{Cr}$ (VI) -adsorption: The aqueous solutions with constant $\mathrm{Cr}$ (VI) concentration $(200 \mathrm{mg} / \mathrm{L})$ were prepared by dissolving $\mathrm{K}_{2} \mathrm{Cr}_{2} \mathrm{O}_{7}$ in deionized water. The $\mathrm{pH}$ values of the solution were adjusted to 5 by hydrochloric acid and sodium hydroxide solution. Cr-removal experiments were then performed by adding $50 \mathrm{mg}$ as-prepared porous hematite into the solutions at room temperature, followed by sampling the solution at defined time intervals for analysis of $\mathrm{Cr}$ content in the solution. The $\mathrm{Cr}$ content was determined on an inductively coupled plasma atomic emission spectrophotometer (ICPAES Atomscan Advantage).

\section{RESULTS AND DISCUSSION}

After the precursor solution was heated at $180^{\circ} \mathrm{C}$ for $2 \mathrm{~h}$, the red powders were collected by a centrifuge. The corresponding x-ray diffraction (XRD) is illustrated in Fig.1. The diffraction peaks match well with the standard values of $\alpha-\mathrm{Fe}_{2} \mathrm{O}_{3}$ (JCPDS NO. 89-2810), indicating that the as-prepared products are $\alpha-\mathrm{Fe}_{2} \mathrm{O}_{3}$ phase.

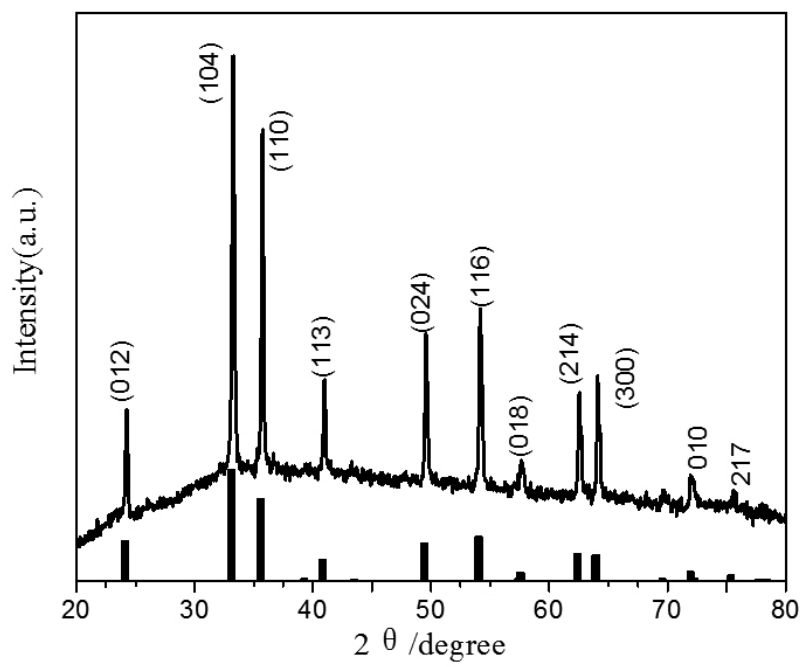

Figure 1. XRD patterns of the as-synthesized and standard $\alpha-\mathrm{Fe}_{2} \mathrm{O}_{3}$ powders (the line spectrum)

Field emission scanning electron microscopic (FESEM) observations have shown that the obtained $\alpha-\mathrm{Fe}_{2} \mathrm{O}_{3}$ crystals is of collective-like morphology with nearly spindle shape, as illustrated in Figure 2A and its inset. 

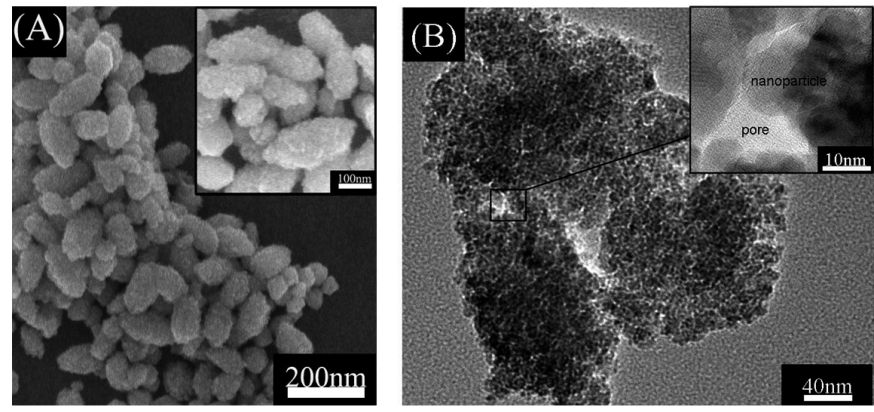

Figure 2. Microstructural examination of $\alpha-\mathrm{Fe}_{2} \mathrm{O}_{3}$ microspindles. (A): low-magnification SEM image (B): low-magnification TEM image (Insets: corresponding high-magnification).

The microstructural examination was further conducted for such $\alpha-\mathrm{Fe}_{2} \mathrm{O}_{3}$ microspindles. Fig. 2B presents the typical TEM image. Under the transmission electron microscopic (TEM) fields, some micropores on the microspindles are found clearly. At the same time, the rough surface of each microspindle was also found. It means that every hematite micrspindle is packed with many ultrafine $\alpha-\mathrm{Fe}_{2} \mathrm{O}_{3}$ nanoparticles from different crystal orientation.

The formation of the hematite could be described in the following reactions.

$$
2 \mathrm{Fe}(\mathrm{OH})_{3} \stackrel{180^{\circ} \mathrm{C}}{\longrightarrow} \alpha-\mathrm{Fe}_{2} \mathrm{O}_{3}+3 \mathrm{H}_{2} \mathrm{O} \uparrow
$$

During heating at $180^{\circ} \mathrm{C}$, the complexes $\mathrm{Fe}(\mathrm{OH})_{3}$ will decompose and form $\alpha-\mathrm{Fe}_{2} \mathrm{O}_{3}$ molecules [reaction (1)]. When the concentration of $\alpha-\mathrm{Fe}_{2} \mathrm{O}_{3}$ molecules was supersaturated, ultrafine $\alpha-\mathrm{Fe}_{2} \mathrm{O}_{3}$ nanoparticles would be formed in the solution by nucleation and growth. Finally, these ultrafine $\alpha-\mathrm{Fe}_{2} \mathrm{O}_{3}$ nanoparticles will be assembled into $\alpha-\mathrm{Fe}_{2} \mathrm{O}_{3}$ microspindles due to reduce the energy of the reaction system.

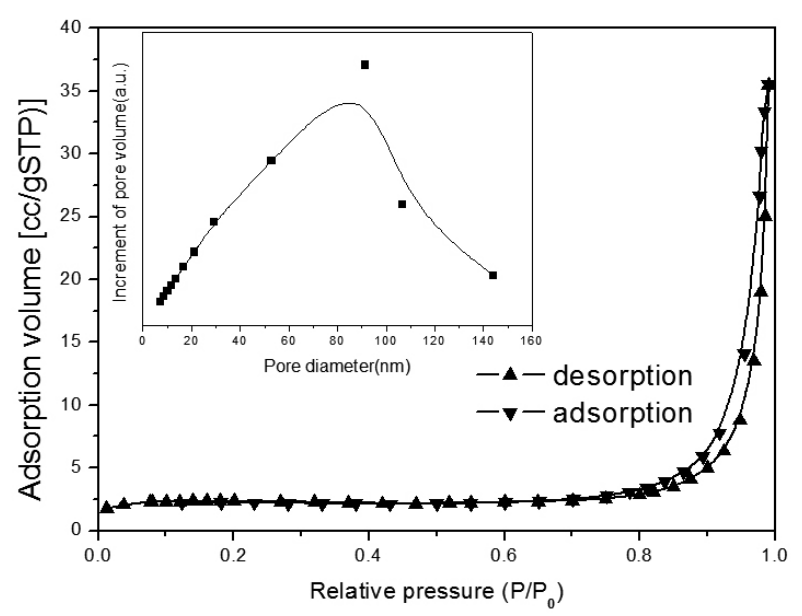

Figure 3. $\mathrm{N}_{2}$ sorption isotherm of the porous hematite microspindles. Inset: pore diameter distribution.

Further, the nitrogen sorption measurement was conducted to evaluate the porous structure and specific surface area for such hematite microspindles. Fig. 3 shows the nitrogen adsorption-desorption isotherm. The specific surface area was thus estimated, by Brunauer-Emmett-Teller (BET) equation [22], to be $78 \mathrm{~m}^{2} / \mathrm{g}$. In addition, the sorption exhibits type IV isotherm and the pore analysis has revealed that the pore sizes in the porous microparticles mainly fall into $60-100 \mathrm{~nm}$, as seen in the inset of Fig.3. As for the abroad pore size distribution around $80 \mathrm{~nm}$ should be mainly contributed from the pileup of the microspindles during $\mathrm{N}_{2}$ sorption measurement.

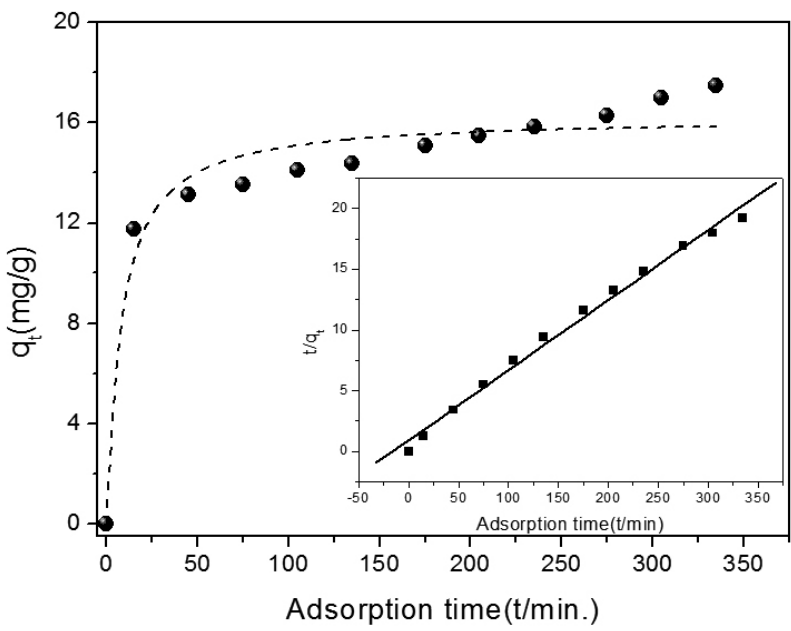

Figure $4 \mathrm{Cr}$-removal as a function of adsorption time in the $100 \mathrm{ml}$ aqueous solution with the initial $\mathrm{Cr}$ (VI) concentration of $200 \mathrm{mg} \mathrm{L}^{-1}$ with $50 \mathrm{mg}$ porous hematite

Fig.4 shows the effect of contact time on the sorbed amounts of Cr (VI) onto porous hematite microspindles in the $100 \mathrm{~mL}$ aqueous solution at room temperature. The pseudo second-order equation (2) $[23,24]$ was used to evaluate the scatter testing data and the nonlinear plot of $q_{t}$ against $t$ was shown as a dash line.

$$
\frac{t}{q_{t}}=\frac{1}{k_{2} q_{e}^{2}}+\frac{1}{q_{e}} t
$$

Where $\mathrm{q}_{\mathrm{e}}(\mathrm{mg} / \mathrm{g})$ is the equilibrium adsorbed amount, $\mathrm{q}_{\mathrm{t}}(\mathrm{mg} / \mathrm{g})$ is the adsorbed amount at any given time $(\mathrm{t}), \mathrm{K}_{2}$ (g/mg.mins) is the equilibrium rate constant of pseudo second-order adsorption.

In the same way, the linear curve-fitting plot of $t / \mathrm{q}_{t}$ versus $t$ gives a straight line as shown in the inset of Fig.4. The correlation coefficient for the linear plot was observed to be close to 1 for the entire adsorption period, which confirmed that the pseudo second-order equation is best suitable for the description of the adsorption kinetic of $\mathrm{Cr}(\mathrm{VI})$ onto $\alpha-\mathrm{Fe}_{2} \mathrm{O}_{3}$ nanomaterials.

The amount of $\mathrm{Cr}(\mathrm{VI})$ sorption on the as-prepared $\alpha-\mathrm{Fe}_{2} \mathrm{O}_{3}$ microspindles is about $15 \mathrm{mg} / \mathrm{g}$. This is much higher than the previously reported values of the other nanomaterials as $\mathrm{Cr}$ (VI)-removal adsorbents, such as polyacrylonitrile/ ferrous chloride composite porous materials $(11.7 \mathrm{mg} / \mathrm{g})[25]$ and magnetite $\mathrm{Fe}_{3} \mathrm{O}_{4}$ nanoparticles $(2.95 \mathrm{mg} / \mathrm{g}$ ) [26]. It is clear from the accuracy of the model that the adsorption kinetic of $\mathrm{Cr}$ (VI) is described by a pseudo second-order chemical reaction and that this reaction is significant in the rate-controlling step. Physical adsorption and chemisorptions may be indistinguishable in certain situations, and in some cases a degree of both types of bonding can be present.

\section{CONCLUSIONS}

In conclusion, this work demonstrates a fast synthesis of spindly hematite $\left(\alpha-\mathrm{Fe}_{2} \mathrm{O}_{3}\right)$ nanomaterials with a porous structure. The proposed method is easy, nontoxic, and reproducible. The as-prepared porous $\alpha-\mathrm{Fe}_{2} \mathrm{O}_{3}$ nanomaterials have higher surface area and can be used to effectively remove the $\mathrm{Cr}(\mathrm{VI})$ ions in wastewater. The calculated model of the adsorption kinetic of $\mathrm{Cr}(\mathrm{VI})$ is described by a pseudo second-order equation.

\section{ACKNOWLEDGEMENTS}

The authors wish to acknowledge with thanks support of this research by the Natural Science foundation of Education Department of Anhui Province (No. KJ2012B148 and KJ2012B154), the Nature Science Foundation of Anhui province (No. 10040606Q53 and 11040606M100) and the Natural Science Foundation of Hefei University (No.12RC02). 


\section{REFERENCES}

[1] Yuanhui Zheng, Yao Cheng, Yuansheng Wang, Feng Bao, Lihua Zhou, Xiaofeng Wei, Yingying Zhang, and Qi Zheng, J. Phys. Chem. B 110, 3093(2006)

[2] Li li Li, Ying Chu, Yang Liu, and Lihong Dong, J. Phys. Chem. C 111, 2123(2007)

[3] Zhengcui Wu, Kuai Yu, Shudong Zhang, and Yi Xie, J. Phys. Chem. C 112, 11307(2008)

[4] Xianluo Hu, Jimmy C. Yu, Jingming Gong, Quan Li, and Guisheng Li, Adv. Mater. 19, 2324(2007)

[5] Changzheng Wu, Ping Yin, Xi Zhu, Chuanzi OuYang, and Yi Xie, $J$. Phys. Chem. B 110, 17806(2006)

[6] Suyuan Zeng, Kaibin Tang, Tanwei Li, Zhenhua Liang, Dong Wang,Yongkun Wang, and Weiwei Zhou, J. Phys. Chem. C 111, 10217(2007)

[7] Kevin Sivula, Radek Zboril, Florian Le Formal, Rosa Robert, Anke Weidenkaff, Jiri Tucek, Jiri Frydrych, and Michael Gra" tzel, J. Am. Chem. Soc. 132, 7436(2010)

[8] Suyuan Zeng, Kaibin Tang, Tanwei Li, Zhenhua Liang, Dong Wang, Yongkun Wang, Yunxia Qi, and Weiwei Zhou, J. Phys. Chem. C 112, 4836(2008)

[9] Xiaoling Wang, S. O. Pehkonen, and Ajay K. Ray, Ind. Eng. Chem. Res. 43, 1665(2004)

[10] L. C. Hsu, S. L. Wang, Y. C. Lin, M. K. Wang, P. N. Chiang, J. C. Liu, W. H. Ku A n, C. C. Chen, and Y. M. T Zou, Environ. Sci. Technol. 44 6202(2010)

[11] Syam K. Prabhakaran, K. Vijayaraghavan, and R. Balasubramanian, Ind. Eng. Chem. Res. 48, 2113(2009)
[12] Inmaculada Ortiz, M. Fresnedo San Roma'n, Sergio M. Corvala'n,and Ana M. Eliceche, Ind. Eng. Chem. Res. 42, 5891(2003)

[13] Yun Qing Xing, Xue Ming Chen, and Da Hui Wang, Environ. Sci. Technol. 41, 1439(2007)

[14] Dongye Zhao, Arup K. SenGupta, and Lori Stewart, Ind. Eng. Chem. Res. 37, 4383(1998)

[15] Yunfeng Xu, Jia Zhang, Guangren Qian, Zhong Ren, Zhi Ping Xu, Yueying Wu, Qiang Liu, and Shizhang Qiao, Ind. Eng. Chem. Res. 49, 2752( 2010)

[16] Zhong, L. S.; Hu, J. S.; Liang, H. P.; Cao, A. M.; Song, W. G.; Wan, L. J. Adv. Mater. 18, 2426(2006)

[17] Yuxin Tang,Yuekun Lai,Dangguo Gong, Kok Hui Goh,Teik Thye Lim, Zhili Dong,and Zhong Chen, Chem. Eur. J. 16, 7704(2010)

[18] Hao Xiang Zhong, Ying Li Ma, Xiao Feng Cao, Xue Tai Chen, and Zi Ling Xue, J. Phys. Chem. C 113, 3461(2009)

[19] Jing Hu, Irene M.C. Lo, and Guohua Chen, Langmuir. 21, 11173(2005)

[20] Sushanta Debnath, Krishna Biswas, and Uday Chand Ghosh, Ind. Eng. Chem. Res. 49, 2031(2010)

[21] Zhi Hui Ai, Ying Cheng, Li Zhi Hang, and Jian Rong Qiu, Environ. Sci. Technol. 42, 6955(2008)

[22] E. P. Barrett, L.G. Joyner, and P.Halenda, J. Am. Chem. Soc. 73, 373(1951)

[23] C. Gerente, V.K.C. Lee, P. Le Clorrec, G. McKay, Crit. Environ. Sci. Technol. 37 41(2007)

[24] Y.S. Ho, J. Hazard. Mater. 136 681(2006)

[25] Y. X. Lin, W. P. Cai, X. Y. Tian, X. G. Liu, G. Z. Wang and C. H. Liang, J. Mater. Chem. 21, 991(2011)

[26] K. M. M. S. Begum, N. Anantharaman, Adsorp. Sci. Tech. 27, 701( 2009) 